\title{
The first GPS-TEC imaging of the space structure of MS wave packets excited by the solar terminator
}

\author{
E. L. Afraimovich ${ }^{1}$, I. K. Edemskiy ${ }^{1}$, S. V. Voeykov ${ }^{1}$, Yu. V. Yasyukevich ${ }^{1}$, and I. V. Zhivetiev ${ }^{2}$ \\ ${ }^{1}$ Institute of Solar-Terrestrial Physics SB RAS, 126a, Lermontov street, Irkutsk, 664033, P.O. Box 291, Russia \\ ${ }^{2}$ Institute of Cosmophysical Research and Radiowave Propagation, FEB RAS, 7, Mirnaya street, Paratunka, Elizovskiy \\ district, Kamchatka region, 684034, Russia
}

Received: 12 September 2008 - Revised: 16 December 2008 - Accepted: 24 March 2009 - Published: 2 April 2009

\begin{abstract}
Using TEC measurements from the dense network of GPS sites GEONET, we have obtained the first GPS-TEC image of the space structure of medium-scale traveling wave packets (MS TWP) excited by the morning solar terminator (ST). We found that ST-generated wave packets have duration of about $1-2 \mathrm{~h}$ and time shift of about $1.5-6 \mathrm{~h}$ after the morning ST appearance at an altitude of $300 \mathrm{~km}$. The TWP wave front extends along the morning ST line with the angular shift of about $20^{\circ}$. The time period and wave-length of ST-generated wave packets are about 10-20 min and 200$300 \mathrm{~km}$, respectively. The velocity of the TWP phase front traveling is of about $300 \mathrm{~m} / \mathrm{s}$. The space image of MS TWP manifests itself in pronounced anisotropy and high coherence over a long distance of about $2000 \mathrm{~km}$.
\end{abstract}

Keywords. Ionosphere (Ionospheric irregularities; Wave propagation; Instruments and techniques)

\section{Introduction}

A lot of investigations have shown that ST motion can generate acoustic-gravity waves (AGW), turbulence and instabilities in the ionosphere plasma (Beer, 1978; Cot and Teitelbaum, 1980; Somsikov, 1987; Somsikov and Ganguly, 1995; Hocke and Schlegel, 1996). It is worth noting that among all sources of gravity waves, the moving ST has a special status, since it is a predictable phenomenon, whose characteristics are well known. If ST is considered to be a stable and repetitive source of AGWs, one can derive information on atmospheric conditions from a response of the medium to this input.

Afraimovich (2008) has recently obtained the first GPS TEC evidence for the wave structure excited by ST mov-

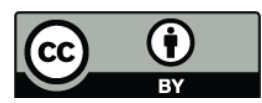

Correspondence to: E. L. Afraimovich (afra@iszf.irk.ru) ing over the USA, Europe, and Japan. The author found two main types of the observed TEC disturbance: large-scale (LS) 60-min variations with amplitude of about $0.5-1$ TECU and medium-scale (MS) 15-min variations with amplitude of about $0.05-0.1$ TECU (TECU is $10^{16} \mathrm{~m}^{-2}$ ). The first type of disturbances was predicted in theoretical investigations and registered earlier using different methods of ionosphere radio sounding. The second type of the observed TEC disturbances is traveling wave packets (TWP) generated when the time derivative of TEC is at its maximum. These TWPs have duration of about $1-2 \mathrm{~h}$ and time shift of about $1.5-2.5 \mathrm{~h}$ after the ST appearance at an altitude of $100 \mathrm{~km}$. These STgenerated wave packets have been found for the first time. The relative amplitude of TEC wave packets is sufficiently low (of about $0.2 \%$ ). This may be the main reason why such a phenomenon has been unknown to date.

To identify ST-generated wave packets it is insufficient to register the TEC time dependence (Afraimovich, 2008). Their spatial structure needs to be determined. But only a dense network of stations can display wave packets. We employ data from the Japanese GPS network GEONET (about 1225 stations in all). At present it is the largest regional GPS network in the world (ftp://terras.gsi.go.jp/data/ GPS_products/).

The goal of this paper is to obtain the first GEONET GPSTEC image of the space structure of ST-generated MS TWP.

\section{Methods of GPS data analyses}

The data we used in this work are available in the standard RINEX format with sampling intervals of $30 \mathrm{~s}$. The standard GPS technology provides means for detecting wave disturbances using phase measurements of the slant TEC $I_{s}$ along the line of sight (LOS) between a GPS site and a satellite. Methods for calculating $I_{s}$ using GPS phase measurements are described in detail in (Calais et al., 2003; Afraimovich

Published by Copernicus Publications on behalf of the European Geosciences Union. 


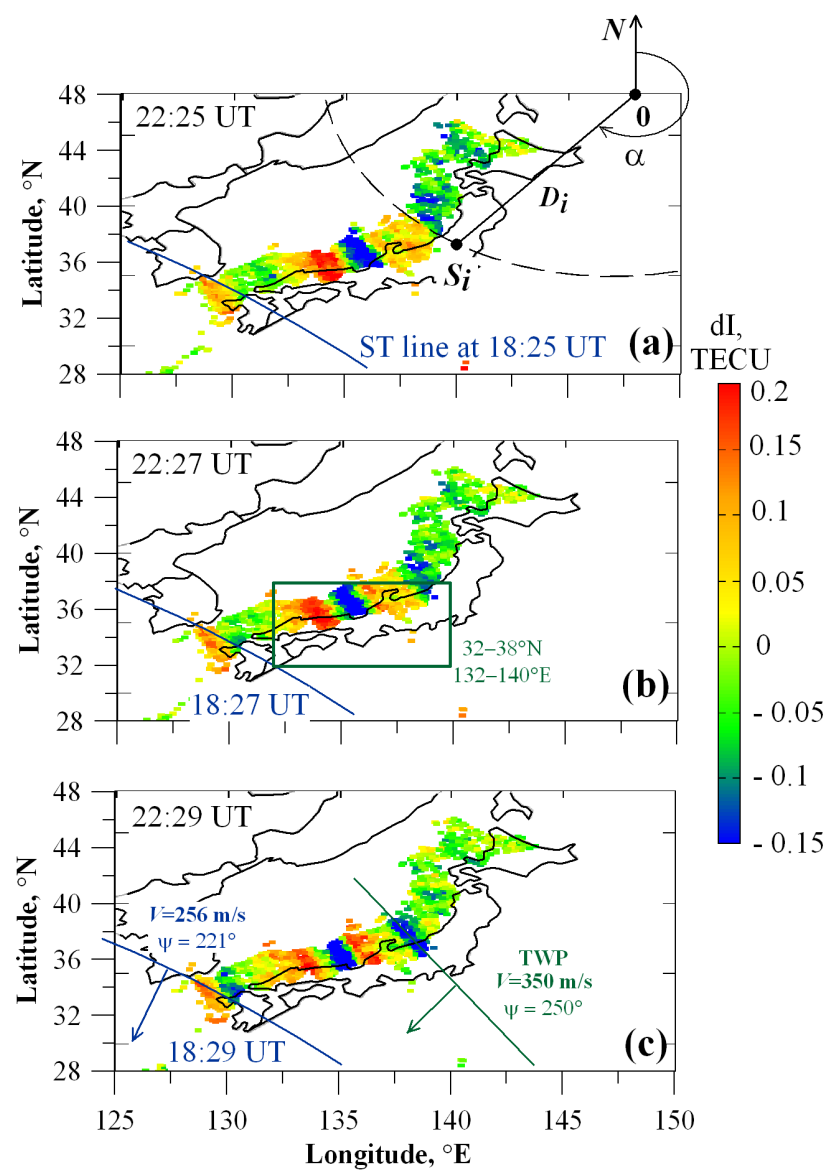

Fig. 1. Geometry of GPS measurements during morning ST motion over Japan on 13 June 2008. Points $\boldsymbol{O}$ and $\boldsymbol{S}_{i}$ mark the relative coordinate centre and the subionospheric point position for LOS between the GPS site with a number $\boldsymbol{i}$ and the selected satellite PRN, respectively; $\boldsymbol{D}_{i}$ is the distance between points $\boldsymbol{O}$ and $\boldsymbol{S}_{i}$ along the great circle arc. 2-D-space distribution of the filtered TEC values $\boldsymbol{d I}(\boldsymbol{\lambda}, \boldsymbol{\phi})$ for PRN10 and for 22:25 UT (a), for 22:27 UT (b); for 22:29 UT (c); solid curves mark the morning ST line at the altitude of $\boldsymbol{H}=300 \mathrm{~km}$ on 18:25 UT (a), 18:27 UT (b), 18:29 UT (c), respectively. Rectangle on the panel (b) marks boundaries of region, in which velocity and direction of TWP propagation was defined. The arrows in Fig. 1c schematically show the wave vector of the TWP (green) and the normal to ST line (dark blue).

et al., 2004). We reproduce here only the final formula for phase measurements:

$I_{s}(t)=\frac{1}{40.308} \frac{f_{1}^{2} f_{2}^{2}}{\left(f_{1}^{2}-f_{2}^{2}\right)}\left[\left(L_{1} \lambda_{1}-L_{2} \lambda_{2}\right)+\right.$ const $\left.+n L\right]$

where $L_{1} \lambda_{1}$ and $L_{2} \lambda_{2}$ are additional paths of the radio signal caused by the phase delay in the ionosphere (m), $L_{1}$ and $L_{2}$ are numbers of phase rotations at the frequencies $f_{1}$ and $f_{2} ; \lambda_{1}$ and $\lambda_{2}$ stand for the corresponding wavelengths, (m); "const" is the unknown initial phase ambiguity, (m); and $n L$ is the error in determining the phase path $(m)$.
To normalize the amplitude of TEC disturbances, we use the transformation of slant TEC into the equivalent vertical value $I(t)$ (Klobuchar, 1986):

$I(t)=I_{S}(t) \times \cos \left[\arcsin \left(\frac{r_{z}}{r_{z}+h_{\max }} \cos \theta_{s}\right)\right]$

where $r_{z}$ is the Earth's radius; $\cos \theta_{s}$ is the LOS elevation to the GPS satellite; $h_{\max }=300$ or $400 \mathrm{~km}$ is the assumed altitude of the ionospheric F2 layer maximum. We neglect the TEC data from low LOS elevation angles (less than $45^{\circ}$ ) to reduce errors due to conversion from slant to vertical TEC.

To smooth the phase measurement noise and to select TEC variations $d I(t)$ in the range of periods (of about $15 \mathrm{~min}$ ) corresponding to the MS TWP one (Afraimovich, 2008), we firstly smooth the initial series with a time window of $2 \mathrm{~min}$ and then remove the linear trend with a window of about $20 \mathrm{~min}$. The filter pass band at an amplitude level of $0.5 \mathrm{ex}-$ tends from 3 to $30 \mathrm{~min}$. Here the amplitude of diurnal and large-scale TEC variations with time periods more than $1 \mathrm{~h}$ damp significantly (Hocke and Schlegel, 1996). A similar procedure of GPS data treatment is usually used by many authors (Calais et al., 2003; Heki and Pink, 2005; Afraimovich et al., 2004; Afraimovich, 2008, etc.).

We use two known forms of the 2-D GPS-TEC image for our presentation of the ST-generated MS TWP space structure. First we apply the diagram "distance-time" $d \boldsymbol{I}(t, D)$ used recently by different authors (Calais et al., 2003; Heki and Pink, 2005). This diagram is calculated in the polar coordinate system (Fig. 1a). Points $\boldsymbol{0}$ and $\boldsymbol{S}_{i}$ mark the relative coordinate centre and the subionospheric point position for LOS between the GPS site with a number $\boldsymbol{i}$ and the selected satellite PRN, respectively; $\boldsymbol{D}_{i}$ is the distance between points $\boldsymbol{O}$ and $\boldsymbol{S}_{i}$ along the great circle arc; $\alpha_{i}$ is the azimuth of direction $\boldsymbol{D}_{i}$, counted off from the northward in a clockwise direction. Here we select the geographic latitude and longitude for points $\boldsymbol{O}\left(48^{\circ} \mathrm{N}\right.$ and $\left.148^{\circ} \mathrm{E}\right)$ outside the GEONET GPS sites field. In this case the most probable value and root-mean-square deviation of $\alpha$ are $236^{\circ}$ and $25^{\circ}$, respectively. Thus as a first approximation we can consider the entire set of GEONET GPS sites to be space-fixed along the most probable direction of $236^{\circ}$ in the narrow azimuth sector of about $25^{\circ}$ over a distance exceeding $2500 \mathrm{~km}$ from the relative coordinate centre $\boldsymbol{0}$. This location of the relative coordinate center was chosen to make more adequate correspondence between distance to each GPS site and its actual location. Also such a location of point $\boldsymbol{O}$ provides more representative demonstration of ST effect on the ionosphere by "distance-time" diagram, because of nearer azimuth values of ST movement direction and direction at GPS site.

We determine the coordinates $x_{i}(t)$ and $y_{i}(t)$ of the subionospheric point $\boldsymbol{S}_{i}$ at an altitude $\boldsymbol{h}_{\max }$ in the topocentric coordinate system with the center in the GPS-site as

$x_{i}(t)=h_{\max } \sin \left(\alpha_{i}(t)\right) \operatorname{ctg}\left(\theta_{i}(t)\right)$

$y_{i}(t)=h_{\max } \cos \left(\alpha_{i}(t)\right) \operatorname{ctg}\left(\theta_{i}(t)\right)$ 


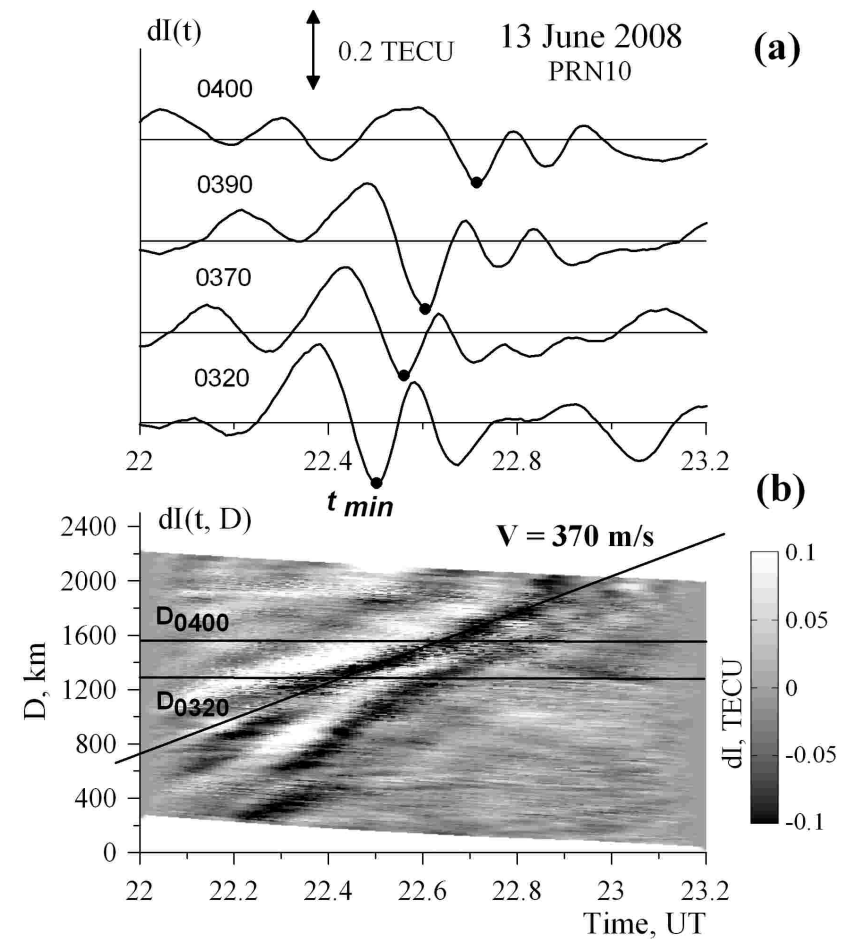

Fig. 2. TEC wave packets on 13 June 2008, for GPS sites located along direction $\mathbf{D}$ over Japan for selected satellite number PRN10. (a) TEC series $\boldsymbol{d I}(\boldsymbol{t})$ filtered in the range of 2-20 min, for selected GPS sites 0320, 0370, 0390 and 0400, (b) the 2-D-dependence of filtered TEC series $\boldsymbol{d I}\left(\boldsymbol{t}, \boldsymbol{D}_{i}\right)$ for all GPS sites $\boldsymbol{S}_{i}$ (total amount is 1081 sites); the horizontal lines $\mathbf{D}_{0320}$ and $\mathbf{D}_{0400}$ mark the distance $\boldsymbol{D}$ from the relative coordinate centre $\boldsymbol{O}$ to GPS/GEONET sites with numbers 0320 and 0400 .

$\alpha_{i}$ is the azimuth; and $\theta_{i}$ is the LOS elevation (Afraimovich et al., 2004). Then we recalculate the topocentric coordinates $x_{i}(t)$ and $y_{i}(t)$ into corresponding values of the latitude $\phi$ and longitude $\lambda$ of the subionospheric point $S_{i}$, calculate the distance $\boldsymbol{D}_{i}$ and then plot the 2-D-diagram "distance-time" $\boldsymbol{d I}(\boldsymbol{t}$, $D$ ) for the selected satellite number PRN. Our approximation is acceptable only for large values of $\theta_{i}$. Then for the minimum $\theta_{i}$ value of about $45^{\circ}$ and $\boldsymbol{h}_{\max }=300 \mathrm{~km}$, the maximum deviation of the subionospheric point $S_{i}$ from GPS site does not exceed $300 \mathrm{~km}$.

In order to study space properties and dynamics of TWP packets in detail we also employ another form of the 2-Dspace distribution of the values of filtered TEC series $\boldsymbol{d} \boldsymbol{I}$ $(\lambda, \phi)$ on the latitude $\phi$ and longitude $\lambda$ for each 30-s TEC counts. Thus we build up the dynamic image of disturbances traveling over Japan in AVI format. The said space-time presentation method of ionosphere disturbances for dense USA GPS networks was used in Tsugawa et al. (2007).

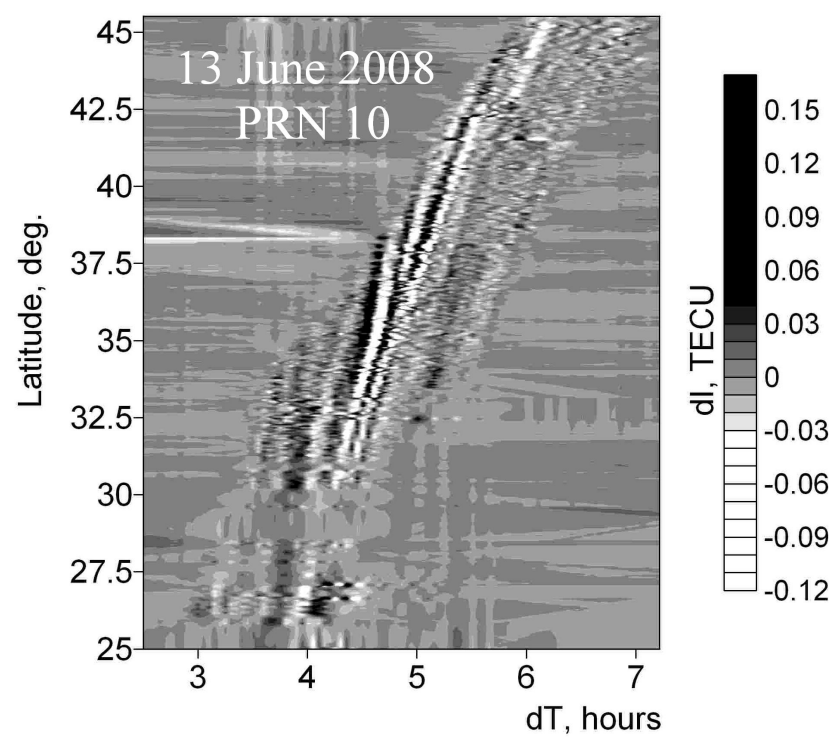

Fig. 3. Filtered TEC series $\boldsymbol{d} \boldsymbol{I}(\boldsymbol{t})$ for PRN10 and for all GPS sites $S_{i}$ in dependence on the latitude $\boldsymbol{\phi}$ and the morning terminator local time $\boldsymbol{d T}$.

\section{Results}

As the first example let's consider geomagnetic quiet day on 13 June 2008 (the $K_{p}$ index varied from 1.0 to 1.7 , http://www.ukssdc.ac.uk/wdcc1/wdc_menu.html). Figure $2 \mathrm{~b}$ presents the 2-D-dependence of TEC series $\boldsymbol{d I}\left(t, \boldsymbol{D}_{i}\right)$, filtered in the range of 2-20 min, for all GEONET sites $S_{i}$ (total amount is 1081 sites) and the satellite PRN10. The amplitude of $\boldsymbol{d} \boldsymbol{I}$ is of about $0.1 \mathrm{TECU}$ (scale for $\boldsymbol{d} \boldsymbol{I}$ is plotted on panel $\mathbf{b}$ at the right). TEC series $\boldsymbol{d I}(\boldsymbol{t})_{i}$ for the selected GPS/GEONET sites 0320, 0370, 0390 and 0400 are shown on panel (a). On panel (b), the horizontal lines $\mathbf{D}_{0320}$ and $\mathbf{D}_{0400}$ mark the distances $\boldsymbol{D}$ from the relative coordinate centre 0 to GPS/GEONET sites with numbers 0320 and 0400 . One can see that TWP travels for $\mathbf{D}_{0320}-\mathbf{D}_{0400}$ distance without a change of shape, but with the time shift (the moment of $\boldsymbol{t}_{\mathrm{min}}$ is marked by thick dots on panel a). Hence TWP travels at apparent propagation velocity $\boldsymbol{V}=\sim 370 \mathrm{~m} / \mathrm{s}$ southwest from the point $\boldsymbol{O}$ over $\boldsymbol{D}=1800 \mathrm{~km}$. The velocity $\boldsymbol{V}$ is defined through the inclination of the line that approximates the dependence of $\boldsymbol{t}_{\min }$ on the distance $\boldsymbol{D}$ (the black line on panel b).

The hypothesis on the connection between the TWP generation and the solar terminator (Afraimovich, 2008) can be tested in the terminator local time (TLT) system: $\boldsymbol{d} \boldsymbol{T}=\boldsymbol{T}_{\mathrm{OBS}}-\boldsymbol{T}_{\mathrm{ST}}$, where $\boldsymbol{T}_{\mathrm{OBS}}$ is the observation time at the given point; $\boldsymbol{T}_{\mathrm{ST}}$ is the arrival time of ST at the altitude of $\boldsymbol{H}$ over this point. In other words, we first transform the latitude and longitude of this point to an arrival time of ST $\boldsymbol{T}_{\mathrm{ST}}$ over this point and then we define the difference $\boldsymbol{d} \boldsymbol{T}$ between the observation time at the given point and the arrival time of ST over this point. A distinctive feature of this approach is 
in excluding certain coordinates of measurement points and considering data on each point in the solar terminator local time only. Figure 3 presents the 2-D-dependence of TEC series $\boldsymbol{d I}(\boldsymbol{d} \boldsymbol{T}, \boldsymbol{\phi})$ for all GEONET sites $\boldsymbol{S}_{i}$ (total amount is 1081 sites) at TLT $\boldsymbol{d} \boldsymbol{T}$ for $\boldsymbol{H}=300 \mathrm{~km}$ and geographic latitude $\phi$ for the selected satellite number PRN10. This dependence has been obtained through the transformation of the time $t$ (UT) into TLT $\boldsymbol{d} \boldsymbol{T}(\mathrm{h})$ from the initial TEC series $\boldsymbol{d I}(\boldsymbol{t}, \boldsymbol{D})$ (Fig. 2b). The TWP 2-D-structure is observed to comprise strong anisotropic waves with the transverse wave-length of about $250 \mathrm{~km}$ and the longitudinal dimension of the phase front exceeding $1500 \mathrm{~km}$. The time delay $\boldsymbol{d} \boldsymbol{T}$ of TWP appearance varies from $2.5 \mathrm{~h}$ at $30^{\circ} \mathrm{N}$ to $6 \mathrm{~h}$ at $45^{\circ} \mathrm{N}$.

It should be noted that the apparent propagation velocity defined by the diagram "distance-time" $d I(t, D)$ corresponds to the velocity projection at the line of primary GPS site distribution (the mean value is $236^{\circ}$, see Sect. 2). To determine proper values of the propagation velocity $\boldsymbol{V}$ and direction $\boldsymbol{\psi}$ of the TWP wave front travel, it is necessary to make 2-D picture of TEC disturbance.

Figure 1 presents 2-D-space distributions of the filtered TEC values $\boldsymbol{d I}(\boldsymbol{\lambda}, \boldsymbol{\phi})$ for 22:25 UT (a), for 22:27 UT (b); for 22:29 UT (c), respectively. Data of each GPS site is represented in the points with coordinates of subionosphere points for LOS between this site and PRN10. Solid curves mark the ST line at the altitude of $\boldsymbol{H}=300 \mathrm{~km}$ for 18:25 UT (a), 18:27 UT (b), 18:29 UT (c), respectively. The preliminary analysis showed that the MS TWP space image manifests itself in pronounced anisotropy and high coherence over a long distance of about $2000 \mathrm{~km}$. The TWP wave front travels predominantly south-westward.

The dynamic image analysis of $d I(\lambda, \phi, t)$ with a time interval of $30 \mathrm{~s}$ gives a precise estimation of the propagation velocity $\boldsymbol{V}$ and azimuth $\boldsymbol{\psi}$ of normal line to the TWP wave front. We employ a method of determining above parameters (SADM-GPS) taking into account the relative motion of subionosphere points (Afraimovich et al., 2004). Various combinations of 3 -sites GPS arrays in the region of $32^{\circ}-$ $38^{\circ} \mathrm{N}, 132^{\circ}-140^{\circ} \mathrm{E}$ (green rectangle in Fig. 1b) for the time interval of 22:00-22:50 UT totaled 191. We select GPS arrays with the base line from $50 \mathrm{~km}$ to $60 \mathrm{~km}$. As deduced from our data, the TWP horizontal propagation velocity $V$ varies from 250 to $450 \mathrm{~m} / \mathrm{s}$, with the most probable value of $350 \mathrm{~m} / \mathrm{s}$. The analysis of the distribution of the TWP traveling azimuth $\psi$ shows a clearly pronounced south-westward direction of TWP displacement of $250^{\circ}$ (r.m.s. is $15^{\circ}$ ). Since the precise azimuth value is close to the preferred direction azimuth of GPS/GEONET location, the precise velocity value is close to $V=370 \mathrm{~m} / \mathrm{s}$ found from the "distancetime" diagram. The mean value of the oscillation time period $(\sim 16 \mathrm{~min})$ corresponds to that of the TWP wave-length of about $300 \mathrm{~km}$.

The arrows in Fig. 1c schematically show the wave vector of the TWP (green) and the normal to the morning ST line (dark blue). It is seen that the TWP wave front extends along the ST line with the angular shift of about $20^{\circ}$. This agrees with the 2-D-distribution of TEC series $\boldsymbol{d I}(\boldsymbol{d} T, \phi)$, Fig. 3. However, the TWP velocity $V$ exceeds that of the ST-line traveling $(256 \mathrm{~m} / \mathrm{s})$.

\section{Conclusion}

We have thus obtained the first GEONET GPS-TEC image of the space structure of MS wave packets excited by the solar terminator and lent support to the validity of the discovery of ST wave packets made by Afraimovich (2008). Our observations also confirm that the ST is a stable and repetitive source of ionospheric wave disturbances (Somsikov, 1987). We found that ST-generated wave packets have duration of about $1-2 \mathrm{~h}$ and time shift of about $1.5-6 \mathrm{~h}$ after the morning ST appearance at an altitude of $300 \mathrm{~km}$. The TWP wave front extends along the ST line with the angular shift of about $20^{\circ}$. The time period and wave-length of ST-generated wave packets are about $10-20 \mathrm{~min}$ and $100-300 \mathrm{~km}$, respectively. The velocity of the TWP phase front traveling is of about $300 \mathrm{~m} / \mathrm{s}$. The space image of MS TWP manifests itself in pronounced anisotropy and high coherence over a long distance of about $2000 \mathrm{~km}$.

The obtained results do not completely agree with the known mechanisms of the ionosphere irregularity generation and propagation (Somsikov, 1987; Somsikov and Ganguly, 1995) and can stimulate the development of the theory. The question on the source of the observed TEC oscillation is still unclear. It is known that TIDs can propagate without significant attenuation and changes in their shape or loss of their coherence no farther than 3-5 wave-lengths; MS TIDs can propagate no farther than $1000 \mathrm{~km}$ (Francis, 1974). The second problem is the time delay of wave packet appearance after the ST passage. The delay may be determined by the correlation between the time damping of MS AGW and the dynamics of TEC growing under different geophysical conditions.

Our results are important for ionospheric irregularity physics development and the transionosphere radio wave propagation modeling. Spatial-temporal features and modeling of MS TWP are the aims of our future investigations.

Acknowledgements. The authors thank E. Calais, J. M. Forbes and J. D. Huba for their interest to this work. We acknowledge the GEONET scientific group for providing GPS data used in this study. Our work was supported by the SB RAS and FEB RAS collaboration project N 3.24, the RFBR-GFEN grant N 06-05-39026 and RFBR grant 07-05-00127, and INTAS Ref. Nr 06-1000013-8823.

Topical Editor M. Pinnock thanks two anonymous referees for their help in evaluating this paper.

\section{References}

Afraimovich, E. L., Astafieva, E. I., and Voyeikov, S. V.: Isolated ionospheric disturbances as deduced from global GPS network, 
Ann. Geophys., 22, 47-62, 2004,

http://www.ann-geophys.net/22/47/2004/.

Afraimovich, E. L.: First GPS-TEC evidence of wave structure excited by solar terminator, Earth, Planets Space, 60, 895-900, 2008.

Beer, T.: On atmospheric wave generation by the terminator, Planet. Space Sci., 26, 185-189, 1978.

Calais, E., Haase, J. S., and Minster, J. B.: Detection of ionospheric perturbations using a dense GPS array in Southern California, Geophys. Res. Lett., 30(12), 1628, doi:10.1029/2003GL017708, 2003.

Cot, C. and Teitelbaum, H.: Generated of the gravity waves by inhomogeneous heating of the atmosphere, J. Atmos. Terr. Phys., 42(9/10), 877-883, 1980.

Francis, S. H.: A theory of medium-scale traveling ionospheric disturbances, J. Geophys. Res., 79, 5245-5259, 1974.

Hocke, K. and Schlegel, K.: A review of atmospheric gravity waves and travelling ionospheric disturbances: 19821995, Ann. Geophys., 14, 917-940, 1996, http://www.anngeophys.net/14/917/1996/.
Heki, K. and Ping, J.: Directivity and apparent velocity of the coseismic ionospheric disturbances observed with a dense GPS array, Earth Planet Sci. Lett., 236, 845-855, 2005.

Klobuchar, J. A.: Ionospheric time-delay algorithm for singlefrequency GPS users, IEEE Transactions on Aerospace and Electronics System, 23(3), 325-331, 1986.

Somsikov, V. M.: A spherical model of wave generation in atmosphere by solar terminator, J. Atmos. Terr. Phys., 49(5), 433-438, 1987.

Somsikov, V. M. and Ganguly, B.: On the mechanism of formation of atmospheric inhomogeneties in the solar terminator region, J. Atmos. Terr. Phys., 57, 75-83, 1995.

Tsugawa, T., Otsuka, Y., Coster, A. J., and Saito, A.: Mediumscale traveling ionospheric disturbances detected with dense and wide TEC maps over North America, Geophys. Res. Lett., 34, L22101, doi:10.1029/2007GL031663, 2007. 